\title{
Bacterial Spectrum and Antimicrobial Resistance Pattern in Cancer Patients with Febrile Neutropenia
}

\author{
Hassan Ali Vahedian-Ardakani ${ }^{1}$, Mansour Moghimi², Mohammad Shayestehpour ${ }^{3,4}$, \\ Masoud Doosti ${ }^{5}$, Nakisa Amid ${ }^{6 *}$
}

\begin{abstract}
Background: Bacterial bloodstream infections are one of the most common complications in cancer patients under treatment. Bacteremia in these patients is a medical crisis that needs antibiotic treatment. The aim of this study was to determine bacterial spectrum and antimicrobial resistance pattern in febrile neutropenic cancer patients. Methods: In this prospective study, 212 cancer patients with febrile neutropenia who were referred to Shahid Sadoughi hospital in Yazd from 2012 to 2015 were participated. Bacterial pathogens isolated by the BACTEC media and antimicrobial susceptibility tests performed according to Clinical and Laboratory Standards Institute (CLSI) guidelines. Results: The mean age of patients was $43.5 \pm 24.98$ years old. Out of 212 participants, $62.3 \%(132 / 212)$ were suffering from hematologic malignancies, and $37.7 \%(80 / 212)$ had solid tumors. Gram-negative bacteria were the predominant microorganisms $(84.9 \%)$. E.coli was the most frequently isolated pathogen $(38.68 \%)$, followed by Klebsiella $(14.15 \%)$ and Acinetobacter species (11.32\%). In addition, Staphylococcus epidermidis was the most common isolated Gram-positive bacteria (8.5\%). Gram-negative bacteria were susceptible to ciprofloxacin with a response range of $53.7 \%$ to $100 \%$. The majority of E.coli isolates were sensitive to ceftazidime (87.8\%) and were resistance to Co-trimoxazole (15.8\%). Klebsiella isolates were 100\% susceptible to cephalosporins, meropenem and imipenem. Conclusion: The majority of bacterial pathogens were resistance to various antibiotics. Judicious use of antibiotic therapy can prevent the emergence and spread of antibiotic-resistant Gram-negative bacteria.
\end{abstract}

Keywords: Antibiotic resistance- bacteria- cancer- febrile neutropenia

Asian Pac J Cancer Prev, 20 (5), 1471-1474

\section{Introduction}

Type of treatment and some risk factors in cancer patients reduce elements of the immune systems, such as neutrophils (Rasmy et al., 2016). Neutropenia can lead to developing serious infection and death. The appearance of fever during neutropenia is usually due to the presence of bacteria, fungi or viruses (Gustinetti and Mikulska, 2016a). Febrile neutropenia (FN) is defined as a single oral temperature measurement $\geq 38.3^{\circ} \mathrm{C}$ or a sustained oral temperature $\geq 38^{\circ} \mathrm{C}$ for more than one hour when absolute neutrophil count (ANC) is less than 500 cell/ $\mu \mathrm{L}$ or the reduction of ANC to $500 \mathrm{cell} / \mu \mathrm{L}$ over the subsequent 48 hours (Freifeld et al., 2011; Meidani et al., 2013).

In patients undergoing chemotherapy for solid tumors, febrile neutropenia represents $50 \%$ of deaths, and the administration of antibiotics reduces the mortality rate to 10\% (Boada Burutaran et al., 2015). In the last decades, some significance advances in the prevention and treatment of febrile neutropenia have led to a decline in the death rate, but still it is considered as a serious problem. However, in patients with solid tumors and hematological malignancies, the mortality rates were estimated 5\% and $11 \%$, respectively (Rasmy et al., 2016).

The pattern of bacterial pathogens causing infection in cancer patients with febrile neutropenia has changed over the past decades (Kanamaru and Tatsumi, 2004). Gram-negative bacteria were detected as the main microorganisms in FN in the 1970, but Gram-positive bacteria became the predominant microorganisms in the 1980 (Rasmy et al., 2016). The prevalence of Gram-negative bacteria increased during the last two decades in adult with FN while in pediatric patients, an increase in Gram-positive bacteria was observed (Meidani et al., 2013). In some countries in Asia, such as Malaysia and Pakistan, the same trend was reported. Lee et al., (2011) demonstrated that Gram-negative microorganisms were the predominant pathogens in Asia-Pacific region. Several studies in Iran were reported the Gram-negative bacteria as the most common pathogens (Nejad et

${ }^{1}$ Department of Internal Medicine, ${ }^{2}$ Department of Pathology, School of Medicine, ${ }^{5}$ Infectious and Tropical Diseases Research Center, Shahid Sadoughi University of Medical Sciences, ${ }^{6}$ Aliebn- Abitaleb School of Medicine, Islamic Azad University, Yazd Branch, Yazd, ${ }^{3}$ Department of Microbiology and Immunology, Faculty of Medicine, ${ }^{4}$ Autoimmune Diseases Research Center, Kashan University of Medical Sciences, Kashan, Iran.*For Correspondence: h.vahedian@ssu.ac.ir 
al., 2015), but Meidani et al., (2013) showed that the Gram-positive bacteria were more prevalent.

Differences in the microbial spectrum of different countries show that knowing the pathogens associated with FN in each area is important for better management of febrile neutropenia in patients suffering from cancer (Meidani et al., 2013). In addition, with increasing rate of antibiotic resistance, knowledge about antimicrobial susceptibility and resistance patterns of causative bacteria in each region is necessary. Antimicrobial resistance is a current major international public health concern. In the other hands, reports on the bacterial spectrum and antimicrobial resistance pattern of bacteria from patients with cancer and febrile neutropenia in Iran are inadequate.

The aim of this study was to determine bacterial spectrum and antimicrobial resistance pattern in bacteria isolated from cancer patients with febrile neutropenia in Yazd, Iran.

\section{Materials and Methods}

\section{Patients}

A total of 212 febrile neutropenic cancer patients were enrolled in this prospective study. The participants were randomly selected among the cancer patients referred to Shahid Sadoughi hospital in Yazd, Iran, between 2012 and 2015. The inclusion criteria were cancer, fever $\geq$ $38.3^{\circ} \mathrm{C}$, neutropenia (ANC $\left.<500 \mathrm{cell} / \mu \mathrm{l}\right)$ and positive blood culture for bacteria. Patients who had received antibiotics before admission were excluded from the study. Study objectives and procedures were explained to the participants, and they signed a written informed consent form. The study was approved by the Ethics committee of Shahid Sadoughi University of Medical Sciences. Demographics and clinical characteristics of all study participants were collected using a questionnaire.

\section{Isolation of bacteria and antimicrobial susceptibility testing}

Blood samples were taken for white blood cell count and absolute neutrophil count (ANC). In addition, the blood cultures were performed in BACTEC Plus Aerobic (\&Anaerobic)/ F medium (BD, USA). All bottles were incubated in the Bactec 9240 instrument (BD, USA) for five days according to the manufacturer's recommendations. Positive blood culture bottles were removed from the instrument. The Gram stain was performed on bottles, and a portion of the blood-broth mixture was subcultured onto solid plate media based on the results of the Gram stain. Subsequent isolation and identification of the bacteria was performed using conventional diagnostic methods such as, catalase, oxidase, coagulase, bacitracin, optochin, and CAMP test for gram-positive microorganisms, and oxidase, catalase, and biochemical tests for gram-negative isolates. The susceptibilities of all isolates to 14 different antibiotics were determined using disk diffusion (Kirby-Bauer method (Hudzicki, 2009)) according to Clinical and Laboratory Standards Institute (CLSI) guidelines (Wikler, 2009). Antimicrobial susceptibility tests were conducted on Mueller-Hinton agar medium using commercial antibiotic disks (Padtan
Teb Co, Tehran, Iran). The antibiotic disks used in the current study were cefixime $(5 \mu \mathrm{g})$, cefotaxime $(30 \mu \mathrm{g})$, ceftriaxone $(30 \mu \mathrm{g})$, ceftazidime $(30 \mu \mathrm{g})$, ciprofloxacin $(5 \mu \mathrm{g})$, meropenem $(10 \mu \mathrm{g})$, imipenem $(10 \mu \mathrm{g})$, amikacin $(30 \mu \mathrm{g})$, co-trimoxazole $(25 \mu \mathrm{g})$, gentamicin $(10 \mu \mathrm{g})$, nitrofurantoin $(300 \mu \mathrm{g})$, clindamycin $(2 \mu \mathrm{g})$, vancomycin $(30 \mu \mathrm{g})$, cloxacillin $(1 \mu \mathrm{g})$. E. coli (ATCC 25922) and S. aureus (ATCC 29213) were used as the reference strain for the antibiotic susceptibility tests.

\section{Statistical Analysis}

Statistical analysis of data was performed using SPSS version 19.0 (IBM Corp., Armonk, NY, USA). A correlation between gender, age and resistance to antibiotics was assessed by Chi-square test. The antibiotic resistance between patients with solid cancer and hematologic malignancies compared by Student $t$ test. A $p$-value of less than 0.05 was defined significant for all testes.

\section{Results}

The study population comprised 212 cancer patients with febrile neutropenia (98 men and 114 women). The mean age of the patients was $43.5 \pm 24.98$ years old. Out of 212 participants, $62.3 \%$ (132/212) were suffering from hematologic malignancies, and $37.7 \%(80 / 212)$ had solid tumors. The most common isolated microorganisms from febrile neutropenia cancer patients were gram-negative bacteria (180/212: 84.9\%). Escherichia coli was the most frequently isolated pathogen $(38.68 \%)$, followed by Klebsiella (14.15\%) and Acinetobacter species (11.32\%). The Genus and species of isolated pathogens are shown in Table 1.

No significant correlation was observed between gender, age and resistance to antibiotics under study $(p=0.08)$. Resistance to co-trimoxazole in isolates of patients with hematologic malignancies was more than those bacteria isolated from solid cancer patients $(p=0.002)$. Other antibiotics had the same effect on microorganisms of patients with solid cancer and hematologic malignancies $(\mathrm{p}>0.05)$. Gram-negative bacteria were susceptible to ciprofloxacin with a response range of $53.7 \%$ to $100 \%$. The majority of E.coli isolates

Table 1. Bacterial Pathogens Isolated from Cancer Patients with Febrile Neutropenia

\begin{tabular}{lc}
\hline Pathogens & No (\%) \\
\hline Gram-negative bacteria & $180(84.9)$ \\
Escherichia coli & $82(38.68)$ \\
Klebsiella species & $30(14.15)$ \\
Acinetobacter species & $24(11.32)$ \\
Enterobacter species & $22(10.38)$ \\
Pseudomonas species & $14(6.60)$ \\
Proteus species & $8(3.77)$ \\
Gram-positive bacteria & $32(15.1)$ \\
Stapylococcus aurous & $14(6.60)$ \\
Stapylococcus epidermidis & $18(8.50)$ \\
\hline
\end{tabular}


Table 2. Antibiotic Sensitivity of Pathogens Isolated from Febrile Neutropenic Cancer Patients

\begin{tabular}{lcccccccc}
\hline Pathogen & E.coli & $\begin{array}{c}\text { Klebsiella } \\
\text { spp }\end{array}$ & $\begin{array}{c}\text { Acinetobacter } \\
\text { spp }\end{array}$ & $\begin{array}{c}\text { Enterobacter } \\
\text { spp }\end{array}$ & $\begin{array}{c}\text { Pseudomonas } \\
\text { spp }\end{array}$ & $\begin{array}{c}\text { Proteus } \\
\text { spp }\end{array}$ & $\begin{array}{c}\text { S. aureus } \\
\text { epidermidis }\end{array}$ \\
\hline Antibiotic & & & & & & & & \\
Cefixime & 48.8 & 100 & 0 & 0 & 0 & 0 & 100 & ND \\
Cefotaxime & 70.7 & 100 & 0 & 0 & 0 & 0 & 100 & ND \\
Ceftriaxone & 75.6 & 100 & 0 & 0 & 0 & 0 & 80 & 0 \\
Ceftazidime & 87.8 & 100 & 0 & 9.1 & 42.9 & 0 & 100 & ND \\
Ciprofloxacin & 53.7 & 100 & 100 & 63.6 & 57.1 & 100 & 80 & ND \\
Meropenem & 53.7 & 100 & 100 & 40.0 & 100 & 100 & ND & 0 \\
Imipenem & 63.4 & 100 & 100 & 45.5 & 42.9 & 100 & 20 & ND \\
Amikacin & 70.7 & 6.7 & ND & 100.0 & ND & ND & 0 & ND \\
Co-trimoxazole & 15.8 & 26.7 & ND & 100.0 & ND & ND & 80 & ND \\
Gentamicin & 75.0 & 28.6 & ND & 100.0 & 0.0 & ND & 100 & ND \\
Nitrofurantoin & 84.6 & 24.6 & ND & 100.0 & 0.0 & ND & ND & ND \\
Clindamycin & ND & ND & ND & ND & ND & ND & 100 & 0 \\
Vancomycin & ND & ND & ND & ND & ND & ND & 28.6 & 100 \\
Cloxacillin & ND & ND & ND & ND & ND & ND & 100 & 100 \\
\hline
\end{tabular}

ND, not determined

were sensitive to ceftazidime $(87.8 \%)$ and were resistance to co-trimoxazole $(15.8 \%)$. Klebsiella isolates were $100 \%$ susceptible to all third-generation cephalosporins, meropenem and imipenem. Isolates of S.aureus and S.epidermidis exhibited $100 \%$ in vitro sensitivity to cloxacillin. All S.epidermidis isolates were resistance to clindamycin. The antimicrobial resistance patterns of bacterial pathogens isolated in patients with febrile neutropenia are represented in Table 2.

\section{Discussion}

The present study is the first report of the microbial spectrum and antimicrobial susceptibility profile from febrile neutropenic cancer patients in Yazd, Iran. The result of this study from the blood cultures of cancer patients revealed that Gram-negative bacteria were the predominant causative pathogens of bacteremia in febrile neutropenia patients. This finding is similar to reports before 1986 that Gram-negative bacteria were the most common cause of bacteremia (Nejad et al., 2015). During the last decades, the pattern of bacterial infections in febrile neutropenic patients with cancer has considerably changed. Gram-positive strains became predominant from the mid-1980s to several years ago. However, more recently this trend has shifted again toward Gram-negative strains in some countries (Gustinetti and Mikulska, 2016b).

In this study, Gram-negative bacteria were detected in $84.9 \%$ of patients. In previous studies from 2007 to 2013 , the rate of Gram-negative bacteria in febrile neutropenic cancer patients was 24.7 to $73.9 \%$ (mean $48.7 \%$ ) (Trecarichi and Tumbarello, 2014a). Generally, the result obtained from this study is similar to recent reports that demonstrated the reemergence of Gram-negative bacteria as a major cause of infection in cancer patients (Meidani et al., 2013). However, in contrast with our data, Nejad et al., (2015) reported that $66 \%$ isolated bacteria from febrile nutropenic cancer patients in Kerman, Iran was Gram-positive. Use of intravenous catheter and fluoroquinolone prophylactic treatment for neutropenic cancer patients was increased the rate of Gram-positive bacteria. In study of Meidani et al., (2013) conducted in Isfahan, the prevalence of Gram-positive strains $(56.4 \%)$ in blood culture of cancer patients was more than Gram-negative bacteria. In comparison with our study, this study was conducted on low sample size with only 23 positive blood culture.

In the present study, Escherichia coli was the most prevalent isolated Gram- negative bacteria (38.68\%). Furthermore, many previous studies have found similar finding. In those studies, the means of E.coli isolations from blood culture of cancer patients was $30.8 \%$ (Trecarichi and Tumbarello, 2014b). In contrast with other studies, frequency of Klebsiella isolates (14.15\%) in our study was more than rate of Pseudomonas (6.60\%). Pseudomonas aeruginosa was the second most common isolated bacteria in the majority of studies with a mean frequency of $18.8 \%$ (Trecarichi and Tumbarello, 2014a). In the current study, species of Acinetobacter and Enterobacter were isolated from blood culture with a frequency of $11.32 \%$ and $10.38 \%$, respectively. In addition, Proteus was isolated from 8 patients (3.3\%), while this microorganism has been previously isolated infrequently from cancer patients (Baskaran et al., 2007; Sirkhazi et al., 2014). The gram-positive bacteria were isolated from $15.1 \%$ of our study populations and Staphylococcus epidermidis was the most common isolate $(8.50 \%)$. This finding is consistent with the result of other studies in Iran that were reported S.epidermidis as the most frequently gram-positive bacteria in febrile nutropenic cancer patients (Meidani et al., 2013; Amini et al., 2014; Nejad et al., 2015; Rezaee et al., 2017).

Determination of antimicrobial susceptibility profile of bacterial pathogens in febrile neutropenic patients can be useful in selecting the best antimicrobial agent 
(Almaziny, 2014). E coli resistance to fluoroquinolones is a problem in many countries (Huang et al., 2011) and we also found this phenomenon in our results. In this study, the majority of E.coli isolates were susceptible to cephalosporins especially ceftazidime $(87.8 \%)$. Studies conducted between 2006 and 2013 have reported that more than $95 \%$ of the $E$. coli isolates were susceptible to carbapenems (Trecarichi and Tumbarello, 2014a), but in the present study, less than $65 \%$ of E.coli isolates were sensitive to carbapenems. In our study, Klebsiella isolates were highly sensitive (100\%) to cephalosporins and carbapenems. This finding is consistent with other studies that were reported susceptibility ranging from 90 to $100 \%$ for carbapenems (Trecarichi and Tumbarello, 2014a). We found that none of S.epidermidis isolates were susceptible to clindamycin while, all $S$. aurous isolates $(100 \%)$ showed sensitivity to it. Majority of $S$. aurous isolates were resistance to vancomycin. This finding is incompatible with study conducted in Iran by Meidani et al., (2013). However, sample size in their study was low; therefore, they might have been underestimated the resistance rate. Consistent with several studies, no case of resistance to vancomycin was detected in S.epidermidis isolates. In addition, all gram-positive isolates shown susceptibility to cloxacillin. (Kim et al., 2005; Nejad et al., 2015).

In conclusion, infections due to the Gram-negative bacteria were predominant in cancer patients with febrile neutropenia, and the most common isolate was E.coli. The majority of bacterial isolates were resistance to various antibiotics. Judicious use of antibiotic therapy is necessary to prevent the emergence and spread of antibiotic-resistant Gram-negative bacteria.

\section{Conflict of interest}

The authors declare that they have no conflict of interest in the research.

\section{References}

Almaziny MA-I (2014). Isolation, identification, and profile of antibiotic resistance of bacteria in childhood febrile neutropenia patients. Eur J Exp Biol, 4, 1-6.

Amini S, Hadjibabaie M, Jahangard-Rafsanjani Z, et al (2014). Evaluation of febrile neutropenia in patients undergoing hematopoietic stem cell transplantation. Acta Medica Iranica, 52, 38.

Baskaran ND, Gan GG, Adeeba K, et al (2007). Bacteremia in patients with febrile neutropenia after chemotherapy at a university medical center in Malaysia. Int J Infect Dis, 11, 513-7.

Boada Burutaran M, Guadagna R, Grille S, et al (2015). Results of high-risk neutropenia therapy of hematology-oncology patients in a university hospital in Uruguay. Rev Bras Hematol, 37, 28-33.

Freifeld AG, Bow EJ, Sepkowitz KA, et al (2011). Clinical practice guideline for the use of antimicrobial agents in neutropenic patients with cancer: 2010 update by the infectious diseases society of america. Clin Infect Dis, 52, e56-93.

Gustinetti G, Mikulska M (2016a). Bloodstream infections in neutropenic cancer patients: a practical update. Virulence, 7, 280-97.
Gustinetti G, Mikulska M (2016b). Bloodstream infections in neutropenic cancer patients: A practical update. Virulence, 7, 280-97.

Huang K-P, Wang T-F, Chu S-C, et al (2011). Analysis of pathogens and susceptibility in cancer patients with febrile neutropenia and bacteremia: Experience in a single institution in eastern Taiwan. Tzu Chi Med J, 23, 115-8.

Hudzicki J (2009). Kirby-Bauer disk diffusion susceptibility test protocol. $A S M, \mathbf{1}, 1-16$.

Kanamaru A, Tatsumi Y (2004). Microbiological data for patients with febrile neutropenia. Clin Infect Dis, 39, 7-10.

Kim Y-H, Lee H-D, Hah J-O (2005). Bacteremia in pediatric cancer patients: causative organisms and antibiotic sensitivities. Korean J Pediatr, 48, 619-23.

Lee D-G, Kim S-H, Kim SY, et al (2011). Evidence-based guidelines for empirical therapy of neutropenic fever in Korea. Infect Chem, 43, 285-321.

Meidani M, Bagheri A, Khorvash F (2013). A population-based study of bacterial spectrum in febrile neutropenic patients. Jundishapur J Microbiol, 6, 150-6.

Nejad ZE, Ghafouri E, Farahmandi-Nia Z, et al (2015). Isolation, identification, and profile of antibiotic resistance of bacteria in patients with cancer. Iran J Med Sci, 35, 109-15.

Rasmy A, Amal A, Fotih S, et al (2016). Febrile neutropenia in cancer patient: epidemiology, microbiology, pathophysiology and management. J Cancer Prev Curr Res, 5, 00165.

Rezaee MA, Abdinia B, Delpak A, et al (2017). The microbiologic pattern in pediatric cancer patients with febrile neutropenia and bacteremia: A referral hospital-based study in Northwest of Iran. Iran J Pediatr, 27, 1-9.

Sirkhazi M, Sarriff A, Aziz NA, et al (2014). Bacterial spectrum, isolation sites and susceptibility patterns of pathogens in adult febrile neutropenic cancer patients at a Specialist Hospital in Saudi Arabia. World J Oncol, 5, 196-203.

Trecarichi EM, Tumbarello M (2014a). Antimicrobial-resistant Gram-negative bacteria in febrile neutropenic patients with cancer: current epidemiology and clinical impact. Curr Opin Infect Dis, 27, 200-10.

Wikler M (2009). Clinical and Laboratory Standards Institute. 2009. Performance standards for antimicrobial disk susceptibility tests: approved standard, 10th ed. Clinical and Laboratory Standards Institute, Wayne, PA. 29, pp 1-53.

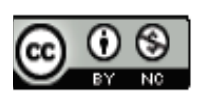

This work is licensed under a Creative Commons AttributionNon Commercial 4.0 International License. 